\title{
Regular exercise, overweight/obesity and sedentary lifestyle cause adaptive changes in thiol-disulfide homeostasis
}

\author{
MEHMET GOL ${ }^{1}$, BEYTULLAH ÖZKAYA ${ }^{1}$, CANER YILDIRIM ${ }^{2}$ and RAMAZAN BAL ${ }^{1}$ \\ ${ }^{1}$ University of Gaziantep, Faculty of Medicine, Department of Physiology, \\ University Avenue, Şehitkamil, 27310 Gaziantep, Turkey \\ ${ }^{2}$ University of Kafkas, Faculty of Medicine, Department of Physiology, Merkez Street, 36100 Merkez, Kars, Turkey \\ Manuscript received on June 4, 2018; accepted for publication on October 29, 2018
}

\begin{abstract}
How to cite: GOL M, ÖZKAYA B, YILDIRIM C AND BAL R. 2019. Regular exercise, overweight/obesity and sedentary lifestyle cause adaptive changes in thiol-disulfide homeostasis. An Acad Bras Cienc 91: e20180547. DOI 10.1590/0001-3765201920180547.
\end{abstract}

\begin{abstract}
Dynamic thiol-disulfide homeostasis is considered to have critical roles in maintenance of physiological functioning. We aimed to reveal whether there is any specific aberration in thiol-disulfide homeostasis in three distinct categories of individuals, including those who 1) exercise regularly (fitness group), 2) have a sedentary lifestyle (sedentary group) and 3) are overweight or obese (overweight/ obese group). 72 male individuals were included in the study, 21 of whom were in fitness group, 28 of whom were overweight or obese and 23 of whom had a sedentary lifestyle. Plasma native thiol (-SH) and total thiol $[(-\mathrm{SH})+(-\mathrm{S}-\mathrm{S}-)]$ levels were quantitatively determined. Total thiol levels in sedentary group were significantly lower than those in overweight/obese $(\mathrm{p}<0.05)$ and fitness groups $(\mathrm{p}<0.001)$. Also, disulfide values in fitness group were significantly higher than those in sedentary and overweight/ obese groups $(\mathrm{p}<0.005, \mathrm{p}<0.05)$. On the other hand, disulfide level, reduced and oxidized thiol ratios and oxidation/reduction ratio in fitness group differed significantly from the other groups $(\mathrm{p}<0.05)$. Thioldisulfide homeostasis varies depending on lifestyle. The results of our study indicate that higher total thiol and disulfide levels are conspicuously distinctive features of thiol-disulfide homeostasis in individuals exercising regularly.
\end{abstract}

Key words: Exercise, obesity, oxidative stress, thiol-disulfide homeostasis.

\section{INTRODUCTION}

Thiols are organosulfur compounds which have sulfydryl groups containing hydrogen and sulfur atoms. Plasma thiols have been more commonly investigated in recent years, because even a mild hyperhomocysteinemia increases the risk of cardiovascular diseases (Andersson et al.

Correspondence to: Beytullah Özkaya

E-mail: beytullahozkaya@gmail.com

ORCid: https://orcid.org/0000-0001-8482-3183
1999). The thiols contribute to the maintenance of homeostasis by participating in synthesis or structure of the proteins, performing some specific tasks in intracellular signalling pathways, having some regulatory roles in some parts of the cell cycle, acting as a catalyst in chemical reactions and forming compounds with metalic elements. Also, they occupy an important place in antioxidant defense system by not only participating in structure of antioxidant enzymes such as thioredoxin, 
glutaredoxin or protein disulfide isomerase but also taking an active role in redox reactions individually (Kiziltunc et al. 2016).

The thiol compounds that primarily constitute the plasma thiols are protein thiols, notably albumin along with low molecular weight thiols, such as cystein, cysteinylglisin, homocystein, glutathione or $\gamma$-glutamylcystein and hydrogen sulfide which is actually a byproduct of transsulfuration reactions. In case of oxidative or nitrosative stress, cystein residues on plasma proteins form disulfide bonds. They are also able to form reversible mixed disulfide bonds between protein thiols and low molecular weight thiols. When the oxidative stress is eliminated, the disulfide bonds can be reduced to thiol groups once again so that thiol disulfide homeostasis is maintained (Kundi et al. 2015, Ozler et al. 2016).

An aberration in the thiol-disulfide homeostasis might play a substantial role as a biomarker in progression of some pathophysiologic entities. It has come out that an impairment in the thioldisulfide homeostasis could be a predictive biomarker for acute myocardial infarction (Kundi et al. 2015). In addition, plasma cystein/glutathione (Cys/GSH) level positively correlates with mortality risk in cardiovascular diseases (Patel et al. 2016). In one of the previous studies, plasma native and total thiol levels of keratoconus patients were ascertained as being significantly higher than that of the control group in the same range of age and sex (Gulpamuk et al. 2017). Moreover, it is speculated that a derangement in thiol-disulfide homeostasis is associated wtih pathophysiological progression of some illnesses such as Alzheimer's disease, parkinsonism, romatoid arthritis, kidney diseases and diabetes mellitus (Erel and Neselioglu 2014).

Increased oxygen consumption during exercise leads to a buildup of reactive oxygen species (ROS) (McLeay et al. 2017). If antioxidant systems can't cope with free radicals, oxidative stress comes into existence. Notwithstanding free radical enhancing effects of exercise, antioxidant enzyme levels increase as a natural consequence of exercise (Hollander et al. 2001, Criswell et al. 1993). Exercise has also been shown to increase aerobic capacity (Miyazaki et al. 2001). Nevertheless, as a result of increased ROS production due to a single period of exercise or a cascade of overloaded exercise sessions, the antioxidant capacity falls short of metabolic requirements and cell damage occurs inevitably (Palazzetti et al. 2003). However, exercise in an appropriate frequency and intensity improves antioxidant capacity and provides a higher level of cardiovascular well-being (Radak et al. 2008).

There are studies manifesting that levels of oxidative stress markers increase in obesity. For instance, isoprostane level depicts a positive correlation with body mass index (BMI) (Keaney et al. 2003). It has been also revealed that as BMI and waist circumference increase, levels of urinary 8-epi-prostaglandin-F2 $\alpha$ and serum thiobarbituric acid-reactive substances also increase. It has been also reported that obesity causes a decrease in mRNA expressions and activities of antioxidant enzymes (Furukawa et al. 2004).

Fat accumulation promotes ROS accumulation in cell cultures. NO levels were found to be lower in overweight young adults (Monda et al. 2014). In healthy adults, it is known that the serum IL-6 levels positively correlate with adipose tissue mass (Higdon and Frei 2003). Furthermore, oxidative stress was reduced in obese subjects with exercise, calorie restriction and diet regulation ( $\mathrm{Li}$ et al. 2017).

To spent most of the day by sitting or lying down, i.e. sedentary behaviour, results in a low grade chronic inflammation and ROS accumulation (Carter et al. 2017). Sedentary behaviour, actually includes various daily life habits, is defined as spending most of the day time sitting or lying down and not having physical activity to increase 
energy expenditure. Lipit peroxidation and protein carbonylation products accumulate in the absence of adequate physical activity (Laufs et al. 2005, Gratas-Delamarche et al. 2014).

Assessment of thiol-disulfide homeostasis is a new biochemical approach to demonstrate plasma oxidative stress level and antioxidant capacity. Indeed, the cysteine residues on peptides or proteins are momentous redox sensors. Furthermore, disulfides or thiol oxidation intermediates are capable of modifying proteins post-translationally or regulating protein function by adjusting folding and structure of proteins. Steady-state levels of plasma thiol-disulfide homeostasis is supplied as a consequens of several processes occurring simultaneously, such as the thiol-disulfide exchange reactions, oxidation of thiol groups by ROS, enzymatic extracellular degradation of glutathione, intracellular metabolism and transport of thiol containing molecules between cells and plasma. These interactions turn the thiol-disulfide homeostasis into an insistent and responsive living compensatory network which effectively perceives redox alterations and are able to potentially provide a diagnostic or prognostic assessment in case of certain aberrations in itself. In this study, redox status and antioxidant capacities in three distinct categories of subjects, including those who exercise regularly, have a sedentary lifestyle and are overweight or obese, were compared by examining thiol-disulfide homeostasis for the first time in literature.

\section{MATERIALS AND METHODS}

This study was carried out on 72 male individuals recruited from Higher School of Physical Education and Sports, Gaziantep University, between June and December 2015. The study started with the approval of Gaziantep University clinical research ethics committee (date: 11.09.2017, protocol number: 2017/313). Of 72 individuals, 21 were in fitness group, 28 were overweight or obese and 23 had a sedentary lifestyle. Demographic characteristics of the groups are illustrated in Table I. The participants were categorized as being overweight/obese or on normal weight by calculating BMI. Those subjects included in overweight/obese group were selected from individuals whose BMI were higher than 25. Besides, overweight or obese subjects had no additional morbidites such as cardiovascular disease or renal diseases, diabetes mellitus, hypertension, hyperlipidemia or autoimmune disorders. Everyday life habits of overweight/ obese group were questioned. They were not doing regular exercise since years. They had a routine life style in which all of them were going to work with a vehicle and spending at least four hours of a day by sitting in front of a worktable, in a bureau. They did not have a exercise program in their daily lives at all such as, swimming, riding bicycle, playing football, basketball etc. In weekends, they usually had social activities like having a picnic, going to the cinema or going out to dinner. Individulas in fitness group had a BMI between 18 and 25 and were following a regular exercise program since at least a few years. According to this program, they had three or four exercise sessions regularly in a week and were working out for at least 40 minutes in each session. The heart rate is over $120 /$ min during those training sessions. The participants in fitness groups also did not have any chronic comorbidity. They were all undergraduate students or research assistants in Physical Education Department of Education Faculty in Gaziantep University. On the other hand, individuals in sedentary group were not following any regular exercise program for years and spending at least four hours sitting in a day as it was the case in overweight/obese group. Their BMI index values varied between 18 and 25, as well.

Venous blood samples of $5 \mathrm{ml}$ were collected from all the subjects included in the study after eight hours of fasting period. Tubes were centrifuged at $4000 \mathrm{rpm}$ for 10 minutes at $4{ }^{\circ} \mathrm{C}$. Blood serum that 
TABLE I

Demographic characteristics of the groups are illustrated in the Table.

\begin{tabular}{cccc}
\hline & Sedentary Group $(\mathbf{n = 2 3})$ & Overweight/Obese Group $(\mathbf{n = 2 8})$ & Fitness Group $(\mathbf{n = 2 1})$ \\
\hline Height $(\mathrm{m})$ & $1.74 \pm 0.06^{2}$ & $1.75 \pm 0.06$ & $1.77 \pm 0.06$ \\
Weight $(\mathrm{kg})$ & $71.80 \pm 6.45^{\#}$ & $93.50 \pm 11.36^{*, \Delta}$ & $76.14 \pm 8.45^{\sharp}$ \\
BMI $\left(\mathrm{kg} / \mathrm{m}^{2}\right)$ & $23.46 \pm 1.20^{\#}$ & $30.24 \pm 2.48^{*}, \Delta$ & $24.19 \pm 2.21^{\sharp}$ \\
Age & $32.52 \pm 4.53$ & $32.60 \pm 3.77$ & $31.84 \pm 3.66$ \\
\hline
\end{tabular}

Statistical values are expressed as mean \pm standard deviation. Following one-way Anova, Tamhane T2 test was used for multiple comparisons.

* $\quad \mathrm{p}<0.05$ as compared to sedentary group.

\# $\quad \mathrm{p}<0.05$ as compared to overweight/obese group.

$\Delta \quad \mathrm{p}<0.05$ as compared to fitness group.

had been obtained after centrifugation is transferred into the eppendorf tubes and stored at $-20{ }^{\circ} \mathrm{C}$.

Plasma native thiol (-SH) and total thiol [(-SH) $+(-\mathrm{S}-\mathrm{S}-)]$ levels were quantitatively determined by a very inexpensive and easy to use biochemical assay method. In this method, briefly, dynamic and reducible disulfide bonds except static or structural ones are turned into the functional thiols by using $\mathrm{NaBH} 4$. The residual NaBH4 compound after aforementioned chemical reaction is completely removed by formaldehyde. The amount of the total thiol content is determined by the Ellman reagent. All the native and reduced thiol groups are treated with 5,5'-dithiobis- (2-nitrobenzoic) acid (DTNB). Dynamic disulfide amount (-S-S) is equal to half of the difference between total and native thiols. In order to calculate disulfide level, the formula given below was used (Altiparmak et al. 2016):

(Serum total thiol - serum native thiol) / 2.

SPSS 24.0 for Windows was used in all statistical analysis. Kolmogorov-Smirnov test was used to determine whether continuous variables were normally distributed for each group. If the statistical variables are normally distributed in their groups, they are presented as mean \pm standard deviation and if not, they are expressed as median in conjunction with first and third quartiles $\left(\mathrm{Q}_{1}-\right.$ $\mathrm{Q}_{3}$ ). One-way ANOVA test was used to compare normally distributed variables in multiple comparisons. Levene test was applied to assess the homogeneity of variances. Post hoc Tukey or Tamhane T2 tests were performed according to the homogeneity of variances for all-pairs multiple comparisons following one-way ANOVA. Independent samples Kruskal Wallis median test was used in multiple comparisons if at least one of the groups did not exhibit normal distribution. For pairwise comparisons, Dunn's test, which gives adjusted $p$ values was applied. Two sided values of $\mathrm{p}<0.05$ were considered as statistically significant.

\section{RESULTS}

The total thiols levels in sedentary group were significantly lower than those in both the overweight/obese group $(\mathrm{p}<0.05)$ and the fitness group $(\mathrm{p}<0.001)$. In addition to that, total thiol levels of fitness groups were significantly higher than those of the overweight/obese group $(p<0.05)$ (Figure 1) (Table II). Although the native thiol values in sedentary group were slightly lower than the other two groups, there was no significant difference among groups $(\mathrm{p}>0.05)$. The disulfide values in fitness group were significantly higher than those in both the sedentary group and the overweight/obese groups $(\mathrm{p}<0.01)$. However, there was no significant difference between the sedentary and the overweight/obese groups ( $p>0.5$ ) (Table II). 
TABLE II

The mean and median values of total thiol, native thiol, disulfide and reduced and oxidized thiol ratio for each group.

\begin{tabular}{|c|c|c|c|c|}
\hline $\begin{array}{l}\text { Biochemical Parameters of } \\
\text { thiol-disulfide homeostasis }\end{array}$ & Sedentary Group* & $\begin{array}{c}\text { Overweight/Obese } \\
\text { Group* }\end{array}$ & Fitness Group* & p value ${ }^{* *}$ \\
\hline Native Thiol $(\mu \mathrm{mol} / \mathrm{L})$ & $335.3(269.8-421,6)$ & $446.8 \pm 135.6$ & $424 \pm 123.2$ & $>0.05$ \\
\hline Total Thiol $(\mu \mathrm{mol} / \mathrm{L})$ & $401.6(382.9-505.4)$ & $543.7 \pm 114$ & $628.5 \pm 101.6$ & $<0.01$ \\
\hline Disulfide $(\mu \mathrm{mol} / \mathrm{L})$ & $20.4(15.4-48.6)$ & $32.6(14.4-69.7)$ & $102.2 \pm 77$ & $<0.01$ \\
\hline Reduced Thiol Ratio ${ }^{1}(\%)$ & $87.57(74.79-95.03)$ & $86.3(71.79-95.45)$ & $68.81 \pm 20.28$ & $<0.05$ \\
\hline Oxidized Thiol Ratio ${ }^{2}(\%)$ & $5.87(2.54-12.26)$ & $6.84(2.32-14.1)$ & $15.59 \pm 10.1$ & $<0.05$ \\
\hline Oxidation/Reduction Ratio ${ }^{3}(\%)$ & $6.66(2.65-16.06)$ & $7.93(2.46-19.04)$ & $22.61(11.61-41)$ & $<0.05$ \\
\hline \multicolumn{5}{|c|}{$\begin{array}{l}\text { 1. }[(-\mathrm{SH}) /(-\mathrm{SH}+-\mathrm{S}-\mathrm{S}-)] \times 100 \\
\text { 2. }[(-\mathrm{S}-\mathrm{S}-) /(-\mathrm{SH}+-\mathrm{S}-\mathrm{S}-)] \times 100 \\
\text { 3. }[(-\mathrm{S}-\mathrm{S}-) /(-\mathrm{SH})] \times 100 \text {, redox status of the thiol disulfide homeostasis. } \\
\text { The disulfide, reduced thiol, the oxidized thiol values and the oxidation/reduction ratio of the fitness group was markedly different } \\
\text { from the other groups, while there was no significant difference between the sedentary and overweight/obese group. } \\
\text { * Provided that the continuous variables are normally distributed in a specific group, the } \\
\text { descriptive statistical value of that group is expressed as mean } \pm \text { standard deviation. If the variables don't exhibit a normal } \\
\text { distribution, it is expressed as median } \pm 25 \%-75 \% \text { percentile ranks. } \\
\text { **P values in Kruskal Wallis test. If there is a significant difference between any two of three groups, pairwise comparison test } \\
\text { (Dunn's test) was applied for all - pairs multiple comparisons. }\end{array}$} \\
\hline
\end{tabular}

On the other hand, both oxidized and reduced thiol ratios also displayed a significant difference among groups. The fitness group exhibited significantly higher oxidized and lower reduced thiol ratios in comparison to the other two groups $(p<0.05$ for both ratios as compared to both the sedentary and overweight/obese groups) (Table II). Nevertheless, there was no markedly difference between the sedentary and overweight/obese groups in terms of the oxidized and reduced thiol ratios. The oxidation/ reduction ratio in fitness group likewise presented a marked difference from both other groups. It is strictly expected that statistical analyses of the oxidized and the reduced thiol ratios and oxidation/ reduction ratio should be consistent among groups. For instance, while oxidized thiol ratios were significantly higher in fitness group, reduced thiol ratios were lower in fitness group in present study.

The total thiol level, extensiveness of which is a delicate indicator of antioxidant competency, varied among three groups. We encountered the largest thiol pool in fitness group (Figure 1). The disulfide level, on the other hand, is actually an exquisite measure of oxidative environment in circulation. As illustrated in Figure 2; fitness group has the highest disulfide levels. It is also noteworthy that the higher total thiol and disulfide levels define the fitness group intrinsically and help draw a distinction between the fitness group and the others, as depicted in ROC analyzes (Figures 3 and 4).

\section{DISCUSSION}

Our results, for the first time, indicates that total thiol levels increase with obesity and regular exercise. Also, disulfide levels in fitness group were significantly higher than those in sedentary and overweight/obese groups. Meanwhile, the oxidized thiol ratio was highest in fitness group in harmony with the highest disulfide levels and the lowest reduced thiol ratio. In addition, the fitness group was significantly differ from each other in terms of the oxidation/reduction ratio. 


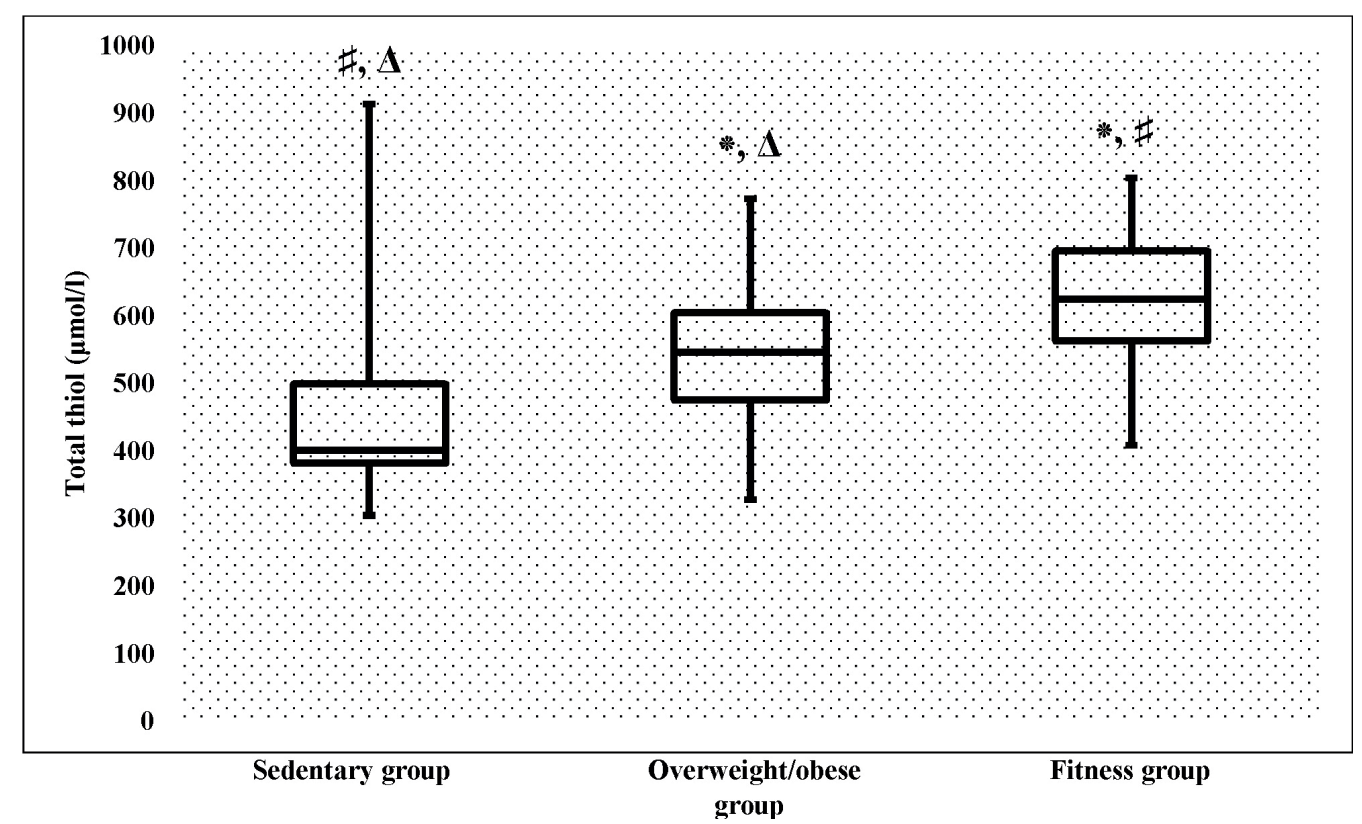

Figure 1 - Total thiol values of groups depicted by using boxplots. Total thiol values in fitness group are significantly higher than those in sedentary and oveweight/obese groups $(p<0.001, p<0.05)$. In addition to that, total thiol levels of overweight/obese group are that of higher than sedentary group $(\mathrm{p}<0.05)$. Independent samples Kruskal Wallis median test was used to compare descriptics of the groups. For pairwise comparison, Dunn's test, which gives adjusted p values was applied.

* $\quad \mathrm{p}<0.05$ as compared to sedentary group.

\# $\quad$ p $<0.05$ as compared to overweight/obese group.

$\Delta \quad \mathrm{p}<0.05$ as compared to fitness group.

It has been shown that people who have been sitting for hours during the daytime display higher adipokine and CRP levels regardless of whether they have been exercising regularly or not. Fibrinogen and CRP levels are consistently higher than normal in overweight individuals with a sedentary lifestyle (Howard et al. 2015). Increased ROS and advanced glycation end products in consequence of prolonged postprandial hyperglycemic period cause endothelial disfunction in sedentaries (Brownlee 2001). In our study, there were no significant differences among groups in terms of native thiol levels. On the other hand, total thiol levels in sedentary group were detected as being significantly lower than those of the other two grops. This finding indicates that total thiol pool, which is a substantial component of the antioxidant defense power of body, is markedly incompetent in sedentary group when compared to the other groups (Table II, Figure 1). This attributes a higher risk of irreversible oxidativenitrosative damage to individuals who internalize a sedentary lifestyle when the level of oxidant substances exceed a reasonable level. Disulfide formation is one of the earliest manifestations of protein oxidation and oxidative stress. We did not detect any significantly elevated disulfide level in sedentary group. However, some previous studies implicated that lipit peroxidation and protein carbonylation products have been increased by virtue of oxidative stress in sedentaries. While the reduced thiol ratio in sedentaries was significantly higher than that of fitness group, the oxidized thiol ratio of sedentaries was significantly lower in comparison to fitness according to outcomes in present study. As a possible reason for this situation, 


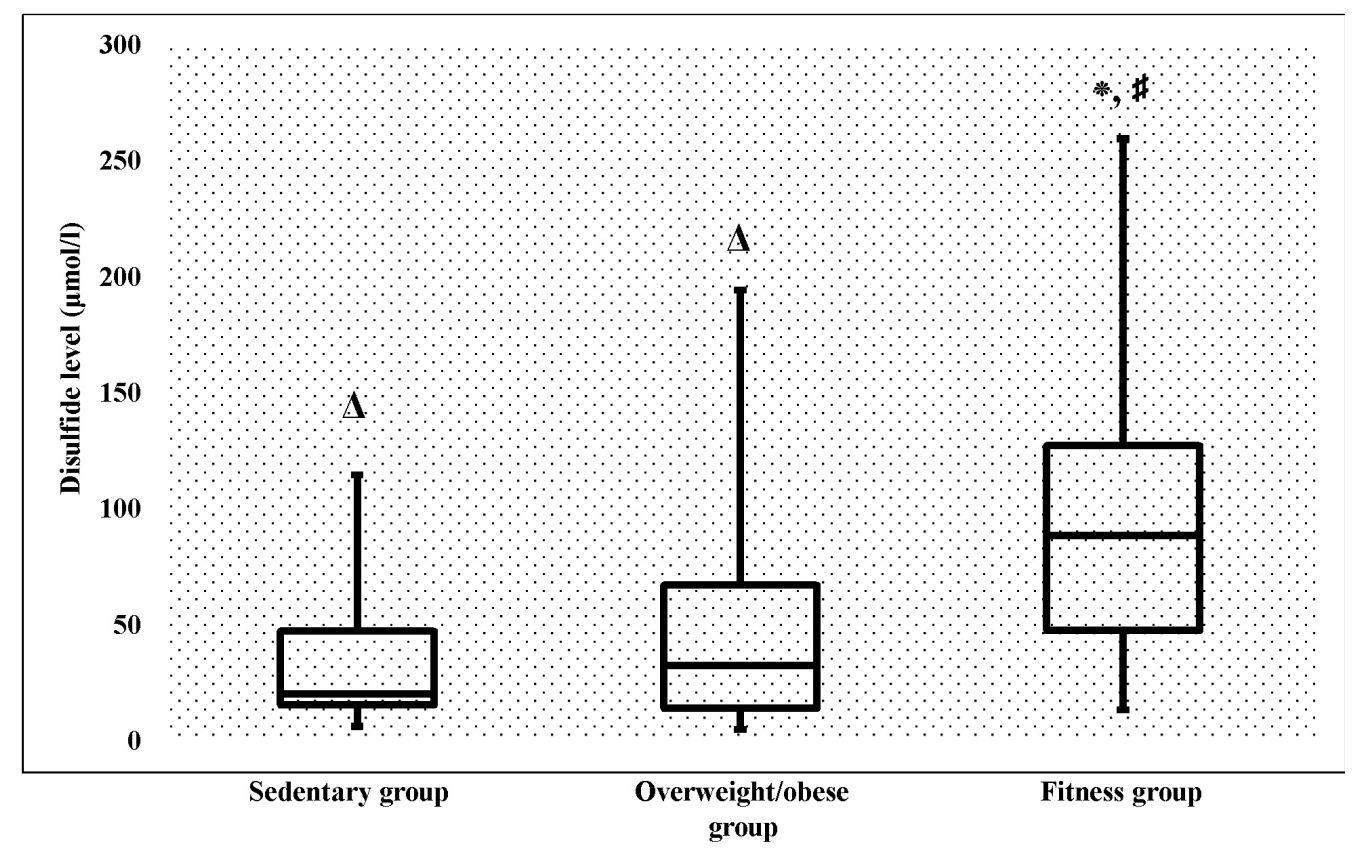

Figure 2 - Disulfide values of groups depicted by using boxplots. Disulfide values in fitness group are significantly higher than those in sedentary group and overweight/obese group $(p<0.005, p<0.05)$. Independent samples Kruskal Wallis median test was used to compare descriptics of the groups. For pairwise comparison, Dunn's test, which gives adjusted p values was applied.

* $\quad \mathrm{p}<0.05$ as compared to sedentary group.

\# $\quad \mathrm{p}<0.05$ as compared to overweight/obese group.

$\Delta \quad \mathrm{p}<0.05$ as compared to fitness group.

we have considered that the level of oxidants mostly range within reasonable bounds in sedentaries so as not to result in oxidative stress, unless individual makes an attempt to have a sudden and challenging training period (Ates et al. 2016, Lushchak 2014). As a consequence, if level of reactive species in metabolism went up for any reason, activation of the physiological antioxidant defense mechanisms and protein thiol modifications would be incompetent (Tebay et al. 2015).

Even if an obese individual does not have any other comorbidity, obesity solely can cause oxidative stress and is associated with increased levels of proinflammatory cytokines. An increase in free fatty acid content of plasma directly results in increased production of ROS in mononuclear and polimorphonuclear cells of the immune system (Tripathy et al. 2003). Warolin et al. (2014) demonstrated a positive correlation between fat proportion of the body and urinary F2-isoprostan level, which is an indicative of oxidative stress. Male C57BL/6J mice fed on a high fatty diet for a long time have exhibited lower GSH/GSSG ratios in gastronecmius muscle and liver (Yuzefovych et al. 2013). Obesity unequivocally causes oxidative stress notwithstanding, there are still some inexplicable parts in obesity induced oxidative stress conception. For instance, in a five years follow-up, increased urinary F2-isoprostan levels were strongly associated with a weight loss and a decrease in fat percentage of the body, especially, in women. This is thought to be due to activation of a particular physiological defense pathway because of obesity induced oxidative stress. Increased level of total thiol in overweight/obese group compared to sedanter group in the present study indicates that the 


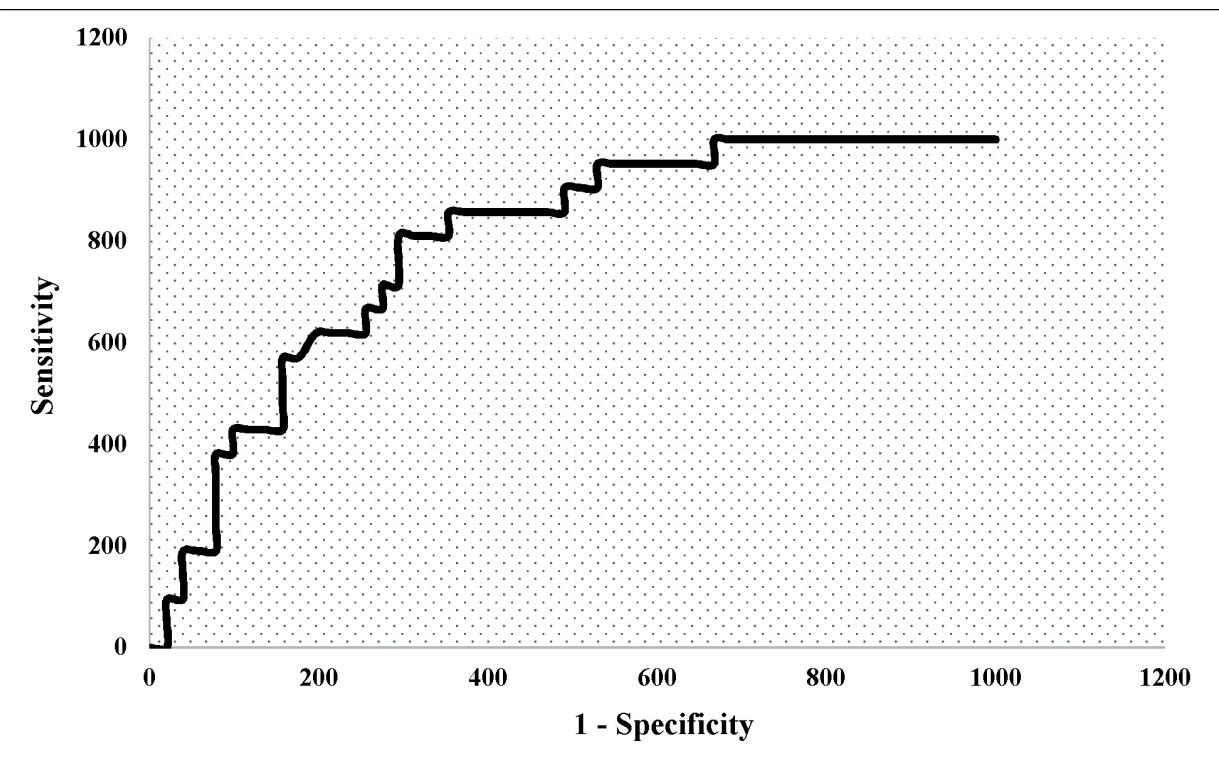

Figure 3 - ROC curve belongs to total thiol values in fitness group is depicted. Area under the curve (AUC): 0.793, Sensitivity: 0.85, Specificity:0.65, 95\% Confidence Interval (CI) lower border:0.687, 95\% Confidence Interval upper border:0.899.

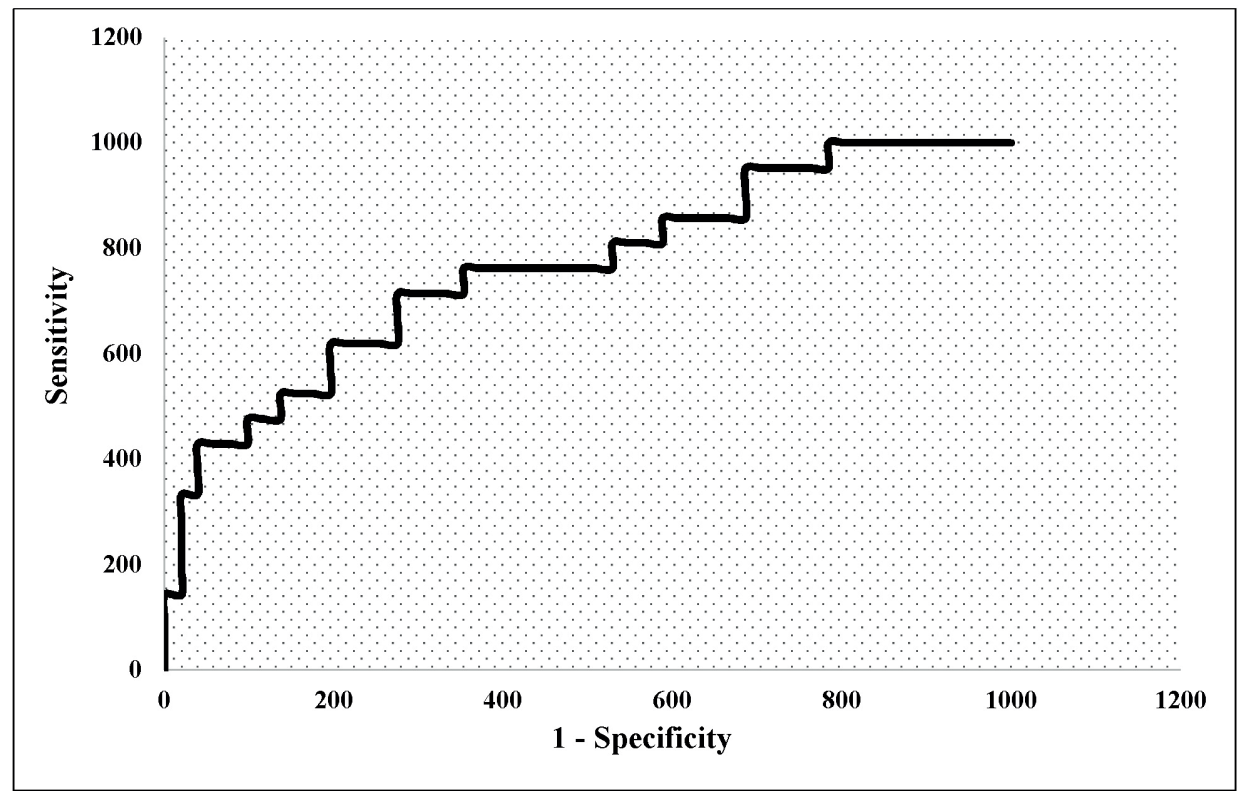

Figure 4 - ROC curve belongs disulfide values in fitness group is depicted. Area under the curve (AUC):0.764, Sensitivity: 0.71, Specificity:0.72, 95\% CI lower border:0.639, 95\% CI upper border:0.889.

thiol pool expands in overweight/obese group. This could be accounted for by previously mentioned physiological defense mechanism against obesity induced oxidative stress (Kanaya et al. 2011). However, disulfide levels were significantly lower in overweight/obese group than those in fitness group in the current study. This suggest that steady state level of oxidant species does not scale up as much in overweight/obese group as it does in fitness group (Table II, Figure 2). 
There is no doubt that exercise promotes oxidative stress. Nevertheless, regular exercise leads to some inventive changes in antioxidant capacity in order to protect cells from harmful effects of oxidative stress and enable the body to cope with increased ROS production (de Lemos et al. 2012). In one of the previous studies, it was revealed that oxygen radical levels in cytoplasmic microenvironment was not elevated after exercise due to upregulation of the antioxidant proteosome complex, despite an increment in reactive carbonyl derivatives as an indication of mitochondrial stress (Radak et al. 2000). In our study, the total thiol levels were significantly higher in fitness group than in the other groups (Table II, Figure 1). So, we deduced that production of reactive species that could be responsible in some physiological mechanisms increased most in fitness group. Correspondingly, this ended in an expansion of total thiol pool as an antioxidant response of the body. This suggests that regular exercise causes some inventive adaptations in thiol-disulfide homeostasis. We concluded that the highest disulfide levels in fitness group is a consequence of the increased free radical production caused by exercise (Figure 2). The higher total thiol and disulfide levels stand out as the defining characteristics of fitness group.

Thiol-disulfide homeostasis varies depending on individual's lifestyle. As illustrated in ROC analyzes, the higher total thiol and disulfide levels distinguish individuals who exercise regularly from the others with a high sensitivity (Figures 3 and 4). On the other hand, the total thiol pool was observed to be expanded, but the disulfide levels did not significantly change in overweight/obese group. In sedentary group, total thiol levels were significantly lower than those of the other groups. In addition, disulfide levels in sedentaries were significantly lower than fitness. This indicates that the steady-state ROS level in sedentary group is lower than that of the other groups.

\section{ACKNOWLEDGMENTS}

The authors would like to thank all participants for their efforts.

\section{AUTHOR CONTRIBUTIONS}

MG presented the hypothesis and wrote the manuscript. BO participated in subject selection and prepared the study design. RB carried out the biochemical analysis. CY carried through the statistical analyses of the acquired data. All authors reviewed the final manuscript.

\section{REFERENCES}

ALTIPARMAK IH ET AL. 2016. Evaluation of Thiol Levels, Thiol/Disulfide Homeostasis and Their Relation with Inflammation in Cardiac Syndrome X. Coron Artery Dis 27(4): 295-301.

ANDERSSON A, LINDGREN A, ARNADOTTIR M, PRYTZ H AND HULTBERG B. 1999. Thiols as a Measure of Plasma Redox Status in Healthy Subjects and in Patients with Renal or Liver Failure. Clin Chem 45(7): 1084-1086. ATES I, OZKAYAR N, INAN B, YILMAZ FM, TOPCUOGLU C, NESELIOGLU S, EREL O, DEDE F AND YILMAZ N. 2016. Dynamic Thiol/Disulfide Homeostasis in Patients with Newly Diagnosed Primary Hypertension. J Am Soc Hypertens 10(2): 159-166.

CARTER S, HARTMAN Y, HOLDER S, THIJSSEN DH AND HOPKINS ND. 2017. Sedentary Behavior and Cardiovascular Disease Risk: Mediating Mechanisms. Exerc Sport Sci Rev 45(2): 80-86.

CRISWELL D, POWERS S, DODD S, LAWLER J, EDWARDS W, RENSHLER K AND GRINTON S. 1993. High Intensity Training-Induced Changes in Skeletal Muscle Antioxidant Enzyme Activity. Med Sci Sports Exerc 25(10): 1135-1140.

DE LEMOS ET, OLIVEIRA J, PINHEIRO JP AND REIS F. 2012. Regular Physical Exercise as a Strategy to Improve Antioxidant and Anti-Inflammatory Status: Benefits in Type 2 Diabetes Mellitus. Oxid Med Cell Longev 2012: 741545.

EREL O AND NESELIOGLU S. 2014. A Novel and Automated Assay for Thiol/Disulfide Homeostasis. Clin Biochem 47(18): 326-332.

FURUKAWA S ET AL. 2004. Increased Oxidative Stress in Obesity and Its Impact on Metabolic Syndrome. J Clin Invest 114(12): 1752-1761.

GRATAS-DELAMARCHE A, DERBRE F, VINCENT S AND CILLARD J. 2014. Physical Inactivity, Insulin Resistance, 
and the Oxidative-Inflammatory Loop. Free Radic Res 48(1): 93-108.

GULPAMUK B, KOC M, KARATEPE MS, YILDIZ A, EREL O, NESELIOGLU S AND YILMAZBAS P. 2017. Novel Assay Assessment of Oxidative Stress Biomarkers in Patients with Keratoconus: Thiol-Disulfide Homeostasis. Curr Eye Res 42(9): 1215-1219.

HIGDON JV AND FREI B. 2003. Obesity and Oxidative Stress: A Direct Link to Cvd? Arterioscler Thromb Vasc Biol 23(3): 365-367.

HOLLANDER J, FIEBIG R, GORE M, OOKAWARA T, OHNO H AND JI LL. 2001. Superoxide Dismutase Gene Expression Is Activated by a Single Bout of Exercise in Rat Skeletal Muscle. Pflugers Arch 442(3): 426-434.

HOWARD BJ, BALKAU B, THORP AA, MAGLIANO DJ, SHAW JE, OWEN N AND DUNSTAN DW. 2015. Associations of Overall Sitting Time and Tv Viewing Time with Fibrinogen and $\mathrm{C}$ Reactive Protein: The Ausdiab Study. Br J Sports Med 49(4): 255-258.

KANAYA AM ET AL. 2011. F2-Isoprostanes and Adiposity in Older Adults. Obesity (Silver Spring) 19(4): 861-867.

KEANEY JUNIOR JF ET AL. 2003. Obesity and Systemic Oxidative Stress: Clinical Correlates of Oxidative Stress in the Framingham Study. Arterioscler Thromb Vasc Biol 23(3): 434-439.

KIZILTUNC E, GOK M, KUNDI H, CETIN M, TOPCUOGLU C, GULKAN B, CICEKCIOGLU H AND ORNEK E. 2016. Plasma Thiols and Thiol-Disulfide Homeostasis in Patients with Isolated Coronary Artery Ectasia. Atherosclerosis 253: 209-213.

KUNDIH,ATES I, KIZILTUNCE, CETINM, CICEKCIOGLU H, NESELIOGLU S, EREL O AND ORNEK E. 2015. A Novel Oxidative Stress Marker in Acute Myocardial Infarction; Thiol/Disulfide Homeostasis. Am J Emerg Med 33(11): 1567-1571.

LAUFS U, WASSMANN S, CZECH T, MUNZEL T, EISENHAUER M, BOHM M AND NICKENIG G. 2005. Physical Inactivity Increases Oxidative Stress, Endothelial Dysfunction, and Atherosclerosis. Arterioscler Thromb Vasc Biol 25(4): 809-814.

LI C, FENG F, XIONG X, LI R AND CHEN N. 2017. Exercise Coupled with Dietary Restriction Reduces Oxidative Stress in Male Adolescents with Obesity. J Sports Sci 35(7): 663-668.

LUSHCHAK VI. 2014. Free Radicals, Reactive Oxygen Species, Oxidative Stress and Its Classification. Chem Biol Interact 224: 164-175.

MCLEAY Y, STANNARD S, HOULTHAM S AND STARCK C. 2017. Dietary Thiols in Exercise: Oxidative Stress Defence, Exercise Performance, and Adaptation. J Int Soc Sports Nutr 14: 12.

MIYAZAKI H, OH-ISHI S, OOKAWARA T, KIZAKI T, TOSHINAI K, HA S, HAGA S, JI LL AND OHNO H.
2001. Strenuous Endurance Training in Humans Reduces Oxidative Stress Following Exhausting Exercise. Eur J Appl Physiol 84(1-2): 1-6.

MONDA M, MESSINA G, SCOGNAMIGLIO I, LOMBARDI A, MARTIN GA, SPERLONGANO P, PORCELLI M, CARAGLIA M AND STIUSO P. 2014. Short-Term Diet and Moderate Exercise in Young Overweight Men Modulate Cardiocyte and Hepatocarcinoma Survival by Oxidative Stress. Oxid Med Cell Longev 2014: 131024.

OZLER S, OZTAS E, CAGLAR AT, UYGUR D, ERGIN M, EREL O AND DANISMAN N. 2016. Thiol/Disulfide Homeostasis in Predicting Adverse Perinatal Outcomes at 24-28 Weeks of Pregnancy in Gestational Diabetes. J Matern Fetal Neonatal Med 29(22): 3699-3704.

PALAZZETTI S, RICHARD MJ, FAVIER A AND MARGARITIS I. 2003. Overloaded Training Increases Exercise-Induced Oxidative Stress and Damage. Can J Appl Physiol 28(4): 588-604.

PATEL RS ET AL. 2016. Novel Biomarker of Oxidative Stress Is Associated with Risk of Death in Patients with Coronary Artery Disease. Circulation 133(4): 361-369.

RADAK Z, CHUNG HY, KOLTAI E, TAYLOR AW AND GOTO S. 2008. Exercise, Oxidative Stress and Hormesis. Ageing Res Rev 7(1): 34-42.

RADAK Z, SASVARI M, NYAKAS C, TAYLOR AW, OHNO H, NAKAMOTO H AND GOTO S. 2000. Regular Training Modulates the Accumulation of Reactive Carbonyl Derivatives in Mitochondrial and Cytosolic Fractions of Rat Skeletal Muscle. Arch Biochem Biophys 383(1): 114-118.

TEBAY LE, ROBERTSON H, DURANT ST, VITALE SR, PENNING TM, DINKOVA-KOSTOVA AT AND HAYES JD. 2015. Mechanisms of Activation of the Transcription Factor Nrf2 by Redox Stressors, Nutrient Cues, and Energy Status and the Pathways through Which It Attenuates Degenerative Disease. Free Radic Biol Med 88: 108-146.

TRIPATHY D, MOHANTY P, DHINDSA S, SYED T, GHANIM H, ALJADA A AND DANDONA P. 2003. Elevation of Free Fatty Acids Induces Inflammation and Impairs Vascular Reactivity in Healthy Subjects. Diabetes 52(12): 2882-2887.

WAROLIN J, COENEN KR, KANTOR JL, WHITAKER LE, WANG L, ACRA SA, ROBERTS LJ II AND BUCHOWSKI MS. 2014. The Relationship of Oxidative Stress, Adiposity and Metabolic Risk Factors in Healthy Black and White American Youth. Pediatr Obes 9(1): 4352.

YUZEFOVYCH LV, MUSIYENKO SI, WILSON GL AND RACHEK LI. 2013. Mitochondrial DNA Damage and Dysfunction, and Oxidative Stress Are Associated with Endoplasmic Reticulum Stress, Protein Degradation and Apoptosis in High Fat Diet-Induced Insulin Resistance Mice. PLoS ONE 8(1): e54059. 